\title{
Prevalence and influence on outcome of HER2/neu, HER3 and NRG1 expression in patients with metastatic colorectal cancer
}

Arndt Stahler ${ }^{a, b}$, Volker Heinemann ${ }^{a, d}$, Jens Neumann ${ }^{b, d}$, Alexander Crispin ${ }^{c}$, Andreas Schalhorn ${ }^{a}$, Sebastian Stintzing ${ }^{a, d}$, Clemens Giessen-Jung ${ }^{a}$, Ludwig Fischer von Weikersthal ${ }^{\mathrm{e}}$, Ursula Vehling-Kaiser ${ }^{f}$, Martina Stauch $^{\mathrm{g}}$, Detlef Quietzsch ${ }^{\mathrm{h}}$, Julian W. Holch ${ }^{\mathrm{a}}$, Stephan Kruger ${ }^{\mathrm{a}}$, Michael Haas ${ }^{\mathrm{a}}$, Marlies Michla ${ }^{\mathrm{a}}$, Jobst von Einem ${ }^{\mathrm{a}}$, Thomas Kirchner ${ }^{\mathrm{b}, \mathrm{d}}$, Andreas Jung ${ }^{\mathrm{b}, \mathrm{d}}$ and Dominik P. Modest ${ }^{a, d}$

\begin{abstract}
Our aim was to explore the impact of the HER2/neu, HER3 receptor as well as their ligands' neuregulin (NRG1) expression on the outcome of patients with metastatic colorectal cancer (mCRC). NRG1, HER2/neu and HER3 expression was evaluated in 208 patients with $\mathrm{mCRC}$ receiving 5-FU/LV plus irinotecan or irinotecan plus oxaliplatin as the first-line treatment. Biomarker expression was correlated with the outcome of patients. NRG1 (low: 192 vs. high: 16), HER2/neu (low: 201 vs. high: 7) and HER3 (low: 69 vs. high: 139) expressions were assessed in 208 patients. High versus low NRG1 expression significantly affected progression-free survival (PFS) [4.7 vs. 8.2 months, hazard ratio (HR): 2.45 ; 95\% confidence interval $(\mathrm{Cl})$ : 1.45-4.13; $P=0.001$ ], but not overall survival (OS) (15.5 vs. 20.7 months, HR: 1.33; 95\% Cl: 0.76-2.35; $P=0.32$ ). High versus low HER3 expression (PFS: 7.1 vs. 8.8 months, HR: 1.11; $95 \% \mathrm{Cl}: 0.82-1.50 ; P=0.50$; OS: 19.8 vs. 21.1 months, HR: $0.95 ; 95 \% \mathrm{Cl}: 0.70-1.30 ; P=0.75)$ and high compared with low HER2/neu expression (PFS: 7.7 vs. 8.0 months, HR: 1.07; $95 \% \mathrm{Cl}: 0.71-1.60 ; P=0.75$; OS: 16.6 vs. 21.1 months, HR: $1.13 ; 95 \% \mathrm{Cl}: 0.75-1.71 ; P=0.57$ ) did not influence outcome. High NRG1 expression was associated with
\end{abstract}

inferior PFS in the FIRE-1 trial. We did not detect a prognostic impact of $H E R 2 / n e u$ and HER3 overexpression in $\mathrm{mCRC}$. The frequency of overexpression was comparable with other studies. Anti-Cancer Drugs

28:717-722 Copyright (c) 2017 Wolters Kluwer Health, Inc. All rights reserved.

Anti-Cancer Drugs 2017, 28:717-722

Keywords: biomarker, HER2/neu, HER3, metastatic colorectal cancer, neuregulin 1

\begin{abstract}
${ }^{a}$ Department of Medicine III, Comprehensive Cancer Centre, University Hospital Grosshadern, ${ }^{b}$ Department of Pathology, 'Institute of Medical Informatics, Biometry, and Epidemiology, University of Munich, ' ${ }^{\mathrm{d}}$ German Department of Translational Cancer Research (DKTK), German Cancer Research Centre (DKFZ), Heidelberg, ${ }^{\mathrm{e}}$ Klinikum St Marien, Oncology, Amberg, ${ }^{f}$ Oncological surgery, Landshut, ${ }^{9}$ Centre of ambulatory treatment for oncological and haematological diseases, Kronach and ${ }^{\text {h}}$ Klinikum Chemnitz, Chemnitz, Germany

Correspondence to Dominik P. Modest, MD, Department of Medicine III, Comprehensive Cancer Centre, University Hospital Grosshadern, Marchioninistrasse 15, Munich D-81377, Germany Tel: + 49894400 72208; fax: + 4989440075252 / + 49897095 5256; e-mail: dominik.modest@med.uni-muenchen.de
\end{abstract}

Received 7 March 2017 Revised form accepted 6 April 2017

HER2/neu overexpression as a targetable structure in a subset of patients with KRAS wild-type mCRC [12]. Besides this potential predictive relevance, the unfavourable outcome of colorectal cancer (CRC) patients has been associated with $H E R 2 / n e u$ overexpression in previous analyses $[13,14]$.

Potentially treatment-relevant expression of the HER3 receptor has been reported in several solid cancer types [15]. If neuregulin (NRG1) binds to the $H E R 3$ receptor, $H E R 3$ forms heterodimers with the $H E R 2 / n e u$ receptor [6]. Subsequently, the $P I 3 K-A K T$ and $M A P K$ pathways are activated, which stimulate tumour proliferation $[6,15]$. In the literature, HER3 expression rates in CRG cells range from 34 to $90 \%$ [15]. However, it is not clear if
HER2/neu overexpression was identified as valuable target in subpopulations of breast cancer and gastric cancer [9-11]. The HERACLES trial has further identified 
$H E R 3$ expression is associated with patients outcome in colorectal cancer. HER3 overexpression was reported to be a negative prognostic marker for patients with CRC without distant metastases [16-18].

In addition, previous investigations focused on the role of $N R G 1$ in CRC as being the activating ligand of the HER3 receptor. De Boeck et al. [19] found tumour progression to be highly influenced by bone marrow-derived mesenchymal stem cells releasing NRG1 in vitro and in vivo. Furthermore, two investigations indicated a potential role for predicting lymph node involvement and the occurrence of distant metastases [20-22]. Nevertheless, two cohorts of advanced CRC and one study of a cohort of CRC patients with distant metastases did not confirm effects on outcome [23-25]. Coalteration of HER2/neu and $H E R 3$ expression was found rarely, also without impact on the outcome of patients with CRC [23].

This analysis was designed to confirm the prevalence and prognostic impact of HER2/neu, HER3 and NRG1 expression in a chemotherapy-based study cohort of 208 patients with mCRC (FIRE-1 trial) receiving either $5-\mathrm{FU} / \mathrm{LV}$ plus irinotecan or irinotecan plus oxaliplatin as first-line therapy. To our knowledge, the FIRE-1 trial is the first randomized-controlled trial to investigate the impact of HER2/neu and HER3 receptor overexpression in relation to $N R G 1$ expression [16-18,20-24].

\section{Methods}

\section{Study design and treatment schedule}

FIRE-1 was a multicentre phase III study. The protocol, primary results and characteristics of patients have been published previously [26]. Also, details on the subpopulation evaluable for translational research have been reported [27]. Information on $R A S$ mutation status and EGFR ligand expression was available for the cohort as described previously [27].

\section{Patients}

Our analysis included 208 of 479 patients with available tumour material [27] for the analysis of HER2/neu, HER3 and NRG1 expression.

\section{Ethics}

The trial was conducted in accordance with the Declaration of Helsinki (1996). All patients provided written informed consent to be treated within a clinical trial. This investigation was performed as a retrospective evaluation with the approval of the local ethics committee of the University of Munich (registry-number: 545-11).

\section{End points}

For this manuscript, overall survival (OS) (time from randomization to death), progression-free survival (PFS) (interval between randomization and death or progression) and response rate (WHO classification: complete remission, partial remission, no change, progressive disease) were used to correlate molecular characteristics with the outcome of patients of the FIRE-1 trial.

\section{Immunohistochemistry}

Immunohistochemistry was performed using $5 \mu \mathrm{m}$ whole standard tissue sections of FFPE tumour samples. For the detection of HER2/neu, a prediluted anti-HER2/neu rabbit monoclonal antibody (clone 4B5; Ventana Medical Systems, Oro Valley, Arizona, USA) was used as the primary antibody. The staining was performed on a Ventana Benchmark XT autostainer using the XT UltraView diaminobenzidine kit (Ventana Medical Systems) following the manufacturer's protocols. Staining of HER3 and NRG1 was performed using the Vectastain ABC-Kit Elite Universal detection system (Vector Laboratories, Peterborough, UK). For HER3 immunohistochemistry, a monoclonal rabbit antibody was used as the primary antibody (ab93739; Abcam, Cambridge, UK). $N R G 1$ staining was performed using a polyclonal human antibody (HPA010964; Atlas Antibodies, Stockholm, Sweden).

\section{Scoring of high and low expression, FISH}

As no evident and standardized method for dividing high and low expression of HER2/neu, HER3 and NRG1 existed at the time of evaluation, we used the HER2/neu score of Rüschoff and colleagues in gastric cancer for scoring complete biomarker expression. Therefore, membrane staining was graduated by intensity $(0$ : none, $1+$ : weak, $2+$ : moderate, $3+$ : strong) and percentage of stained tumour cells (Fig. 1). High expression was defined by a percentage of more than $10 \%$ stained tumour cells and at least moderate $(2+$ or $3+)$ membrane staining versus no or weak staining $(0$ or $1+)$ for low expression. In addition, two-colour fluorescence in-situ hybridization (FISH) was performed in patients showing moderate $(2+) H E R 2 /$ neu staining. Chromosome 17 centromere signals (green) as well as HER2 gene signals (red) were counted in at least 20 nuclei of colorectal tumour cells. Thus, a red to green ratio of at least 2 indicated amplification of HER2. Primary tumour slides were evaluated by two independent observers (A.S. and J.N.) using a light microscope. Disagreements $(<5 \%)$ were reviewed together, followed by conclusive judgement.

\section{Statistical analysis}

OS and PFS stratified by the molecular markers were estimated using Kaplan-Meier analysis. Significant differences were evaluated using the log-rank test and Cox regression analysis. Univariate Cox regression was performed in subgroups. The correlation of clinicopathologic parameters with biomarker expression was assessed using the $\chi^{2}$-test and the Fisher exact test for nominal variables. All $P$-values of less than 0.05 (two sided) were considered significant. SPSS PASW 18.0 (SPSS Inc., Chicago, Illinois, USA) was used for statistical analysis. 
Fig. 1
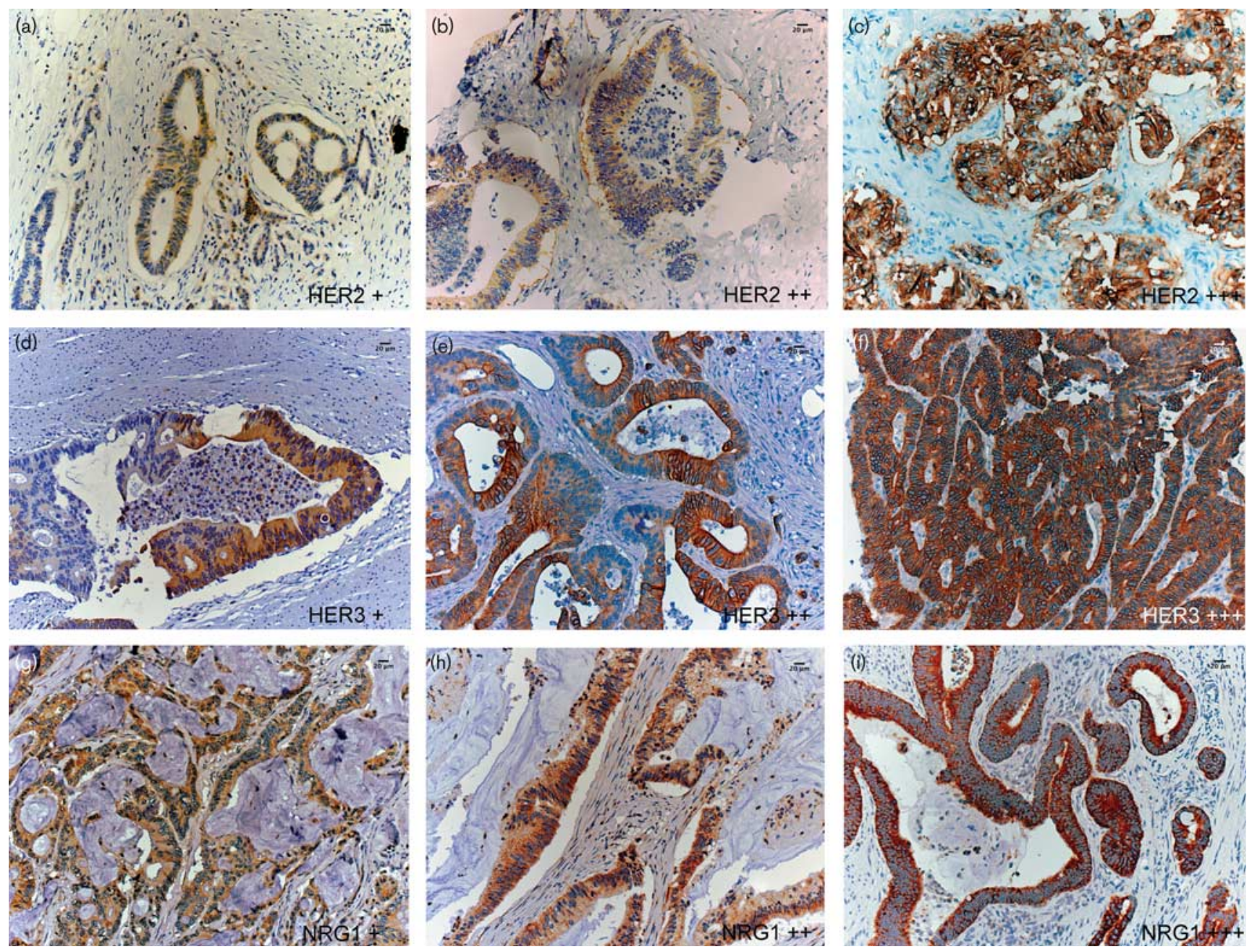

Immunohistochemical staining intensity of $H E R 2 /$ neu expression [ + : weak (a),$++:$ intermediate (b),$+++:$ strong (c)], HER3 expression [ + : weak (d), ++: intermediate (e), +++: strong (f)] and NRG1 expression [ + : weak $(\mathrm{g}),++:$ intermediate $(\mathrm{h}),+++:$ strong (i)]. NRG, neuregulin.

\section{Results}

\section{Study population}

HER2/neu, HER3 and NRG1 analyses were carried out in 208 tumours. Characteristics of the entire patient population and the evaluable subpopulation have been published before [27]. According to baseline and tumour characteristics as well as PFS and OS, the subpopulation was well comparable with the entire study population [27].

\section{Prevalence of high NRG1, HER3 and HER2/neu expression}

Of 208 metastatic colorectal tumours in total, high NRG1 expression was detected in $16(7.7 \%)$ specimens. 139 of 208 tumours $(67 \%)$ were diagnosed to have HER3 overexpression. Twenty-three (11.1\%) patients showed moderate HER2/neu staining. A subsequent FISH analysis, however, showed a missing gene amplification in all of these 23 patients (HER2/neu: chromosome 17 ratio $<2.0)$. Therefore, only strong $(3+)$ stainings in seven (3.3\%) patients were accepted as high HER2/neu expression.

\section{Correlation of NRG1, HER3 and HER2/neu}

A significant correlation of biomarkers with each other could not be detected for NRG1 and HER2/neu $(P=1.00)$ or for NRG1 and HER3 $(P=0.41)$. High HER2/neu expression also did not correlate with HER3 overexpression $(P=0.43$; Table 1$)$.

\section{Association of NRG1, HER3 and HER2/neu expression with RAS mutations and EGFR-ligand expression}

$H E R 3$ overexpression was significantly correlated with the presence of $R A S$ mutations $(P=0.02$; Table 2$)$. HER3 overexpression showed a trend towards an association 
Table 1 Correlation of HER2/neu and HER3 overexpression with NRG1 expression and coalteration of HER2/neu and HER3 expression

Correlation of HER2/neu and HER3 overexpression with NRG1 expression

\begin{tabular}{|c|c|c|c|c|}
\hline & \multicolumn{2}{|c|}{ Neuregulin 1 [ $n(\%)]$} & \multirow[b]{2}{*}{ Total $[n(\%)]$} & \multirow{2}{*}{$\begin{array}{l}P \text { (two } \\
\text { sided) }\end{array}$} \\
\hline & Low & High & & \\
\hline \multicolumn{5}{|c|}{ HER2/neu } \\
\hline Low & $185(89.0)$ & $16(7.7)$ & $201(96.7)$ & 1.00 \\
\hline High & 7 (3.3) & $0(0.0)$ & 7 (3.3) & \\
\hline Total & $192(92.3)$ & $16(7.7)$ & $208(100.0)$ & \\
\hline \multicolumn{5}{|l|}{ HER3 } \\
\hline Low & $62(29.8)$ & $7(3.4)$ & 69 (33.2) & 0.41 \\
\hline High & $130(62.5)$ & $9(4.3)$ & $139(66.8)$ & \\
\hline Total & 192 (92.3) & $16(7.7)$ & $208(100.0)$ & \\
\hline \multicolumn{5}{|c|}{ Coalteration of $H E R 2 / n e u$ and $H E R 3$ expression } \\
\hline & \multicolumn{2}{|c|}{ HER2/neu [n (\%)] } & \multirow[b]{2}{*}{ Total $[n(\%)]$} & \multirow[b]{2}{*}{$P$ (two sided) } \\
\hline & Low & High & & \\
\hline \multicolumn{5}{|l|}{ HER3 } \\
\hline Low & $68(32.7)$ & $1(0.5)$ & 69 (33.2) & 0.43 \\
\hline High & $133(64.0)$ & $6(2.8)$ & $139(66.8)$ & \\
\hline Total & $201(96.7)$ & 7 (3.3) & $208(100.0)$ & \\
\hline
\end{tabular}

$P$-values calculated using the $\chi^{2}$-test. NRG, neuregulin.

Table 2 Correlation of HER3 expression and RAS status

\begin{tabular}{|c|c|c|c|c|}
\hline \multirow[b]{2}{*}{$R A S$} & \multicolumn{2}{|c|}{$H E R 3[n(\%)]$} & \multirow[b]{2}{*}{ Total $[n(\%)]$} & \multirow[b]{2}{*}{$P$ (two sided) } \\
\hline & Low & High & & \\
\hline Wild type & $42(20.2)$ & $59(28.4)$ & $101(48.6)$ & 0.02 \\
\hline Mutation & 27 (12.9) & $80(38.5)$ & $107(51.4)$ & \\
\hline Total & 69 (33.1) & $139(66.9)$ & $208(100.0)$ & \\
\hline
\end{tabular}

$P$-values calculated using the $\chi^{2}$-test.

with high $E R E G$ expression. $(P=0.07)$. All other combinations did not show associations.

\section{Survival analysis}

High versus low NRG1 expression significantly affected PFS (4.7 vs. 8.2 months, hazard ratio: 2.45 ; $95 \%$ confidence interval: $1.45-4.13 ; P=0.001)$, but not $\mathrm{OS}(15.5$ vs. 20.7 months, hazard ratio: 1.33 ; 95\% confidence interval: $0.76-2.35 ; \quad P=0.32)$. HER3 and Her2/neu expression did not influence outcome (Fig 2a-d).

\section{Discussion}

To our knowledge, the FIRE-1 trial is the first randomized-controlled trial to investigate the impact of $H E R 2 / n e u$ and HER3 receptor overexpression in relation to $N R G 1$ expression and $R A S$ status in mCRC [16-18, 20-24]. Previous investigations focused mostly on analysing HER2/neu and HER3 expression in advanced colorectal or rectal tumours with patients receiving adjuvant radiotherapy or chemotherapy $[17,18,20-22]$. The FIRE-1 treatment schedule consisted of 5-FU/LV plus irinotecan or oxaliplatin plus irinotecan as first-line therapy, following a recommended second-line therapy with the respective crossover study regimen. With 208 patients enrolled in the analysis, our trial represents a robust investigation [16-18,20-24].

Evaluation of HER2/neu expression has been established in gastric cancer by Rüschoff et al. [9] using immunohistochemical staining and FISH in intermediate cases. Therefore, we decided to use these validated methods for patients with mCRC in accordance with the literature. HER3 expression was also evaluated by immunohistochemical stainings using a modified Rüschoff semiquantitative scoring system, as in previous investigations, defining overexpression by cytoplasmatic or membrane staining intensity $[16,18,23$, 24]. As scoring of NRG1 expression is not yet standardized for any tumour type [19], we also used an adapted Rüschoff score.

In FIRE-1, 3.3\% of tumours showed high HER2/neu expression. This compares favourably to the average expression rate of HER2/neu in the literature of $\sim 5.0 \%$ $[12,13,23]$. In FIRE-1, 67\% of primary tumours showed high HER3 expression, comparing favourably to recent other cohorts (Seo et al. [23]: 69\%; Lédel et al. [18,22]: 70\%). One trial evaluating transmembrane NRG1 expression in CRC reported a frequency of high expression of $76 \%$, in contrast to $8 \%$ in FIRE-1 [19]. However, a standardized scoring system is missing in this case, which may explain the discrepancy.

We attempted to correlate expression rates of $H E R$ receptors with HER ligands (NRG1) as well as downstream molecules $(R A S)$. Unlike a previous investigation, we could not associate HER3 expression with NRG1 expression [19]. In our investigation, regular simultaneous expression of HER2/neu and HER3 was also not detected, although coexpression of HER2/neu and HER3 has been described in cohort of 364 surgically resected CRC patients [28]. The latter discrepancy might be caused in part by the different clinical backgrounds of patients as well as by the different diagnostic methods used.

By contrast, in our cohort, HER3 expression correlated with $R A S$ mutations, although $H E R 3$ expression could not be associated with KRAS mutations previously [21]. This observation might have resulted from a higher number of patients enrolled in our investigation in addition to an extended analysis of $R A S$ mutations.

In our study, high $N R G 1$ expression led to a significant decrease in PFS. This finding is supported by a previous investigation that also observed significantly worse 5-year PFS in patients with mCRC and high NRG1 expression [19]. Because of the small numbers of patients showing high NRG1 expression, conclusions are limited. The sample size of patients with HER2/neu overexpression in our cohort does not allow for conclusions on the prognostic impact. It is noteworthy that $H E R 3$ overexpression 

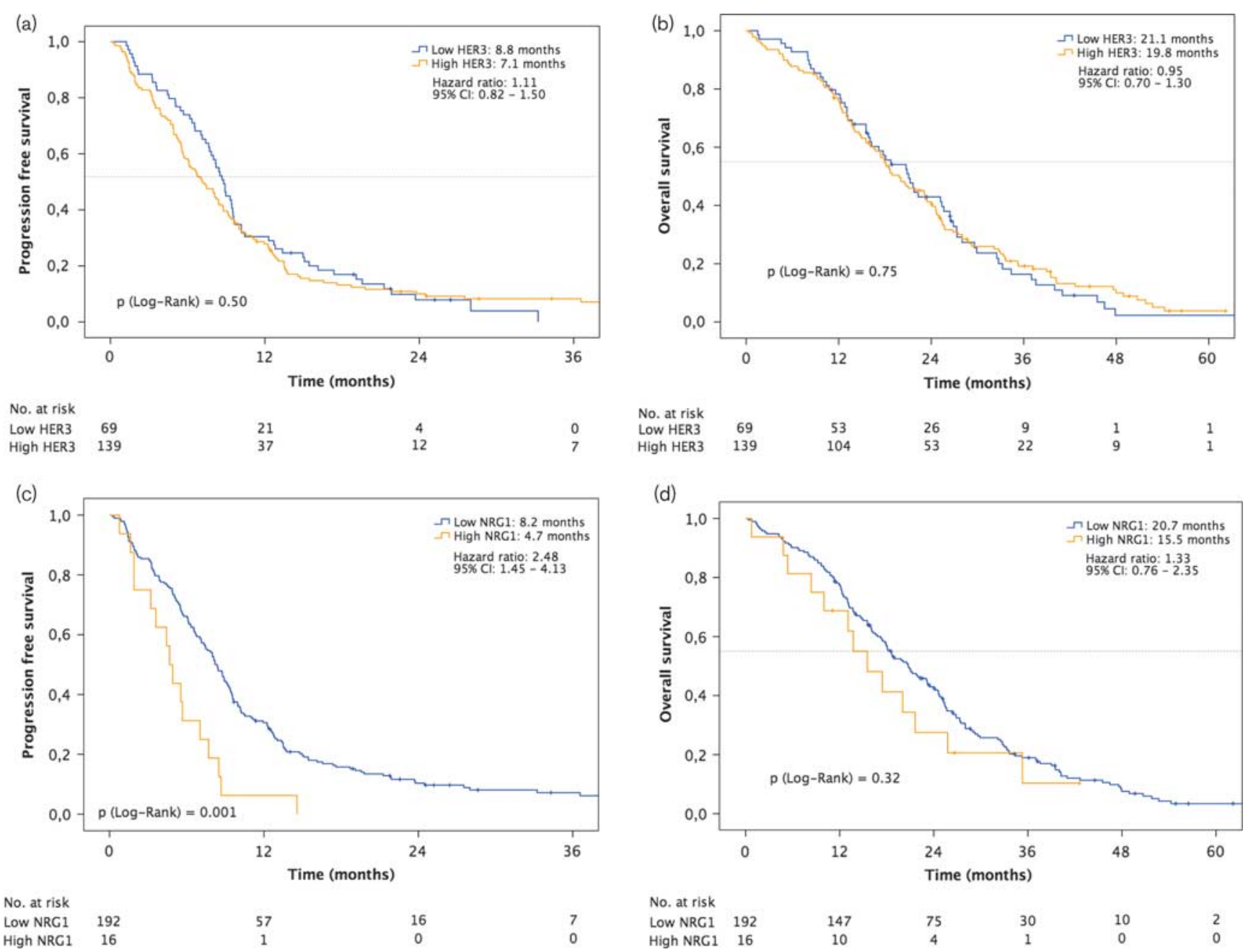

Outcomes according to subgroups in FIRE-1; (a): PFS of patients in FIRE-1 comparing low and high HER3 expression. (b): OS of patients in FIRE-1 comparing low and high HER3 expression. (c): PFS of patients in FIRE-1 comparing low and high NRG1 expression. (d): OS of patients in FIRE-1 comparing low and high NRG1 expression. Cl, confidence interval; NRG, neuregulin; OS, overall survival; PFS, progression-free survival.

was not associated with an unfavourable outcome in FIRE-1, although conflicting data may exist [16-18,24, 28]. However, the small number of patients enrolled in some trials limits conclusions.

Our investigation had several strengths. FIRE-1 was a randomized-controlled trial with irinotecan-based treatment, which had a rather small likelihood of bias in terms of the outcome and follow-up information. Unfortunately, although 208 patients were enrolled in this investigation, which represented a rather robust sample size, numbers in subgroups (NRG1, Her2/neu) became small. It might also be argued that treatment in FIRE-1 does not comply with the latest recommendations. In addition, FIRE-1, as well as previous investigations, lacked a validation collective for a proofof-principle analysis. Therefore, further research is warranted to evaluate prognostic effects.

\section{Conclusion}

A significant unfavourable impact on PFS was observed in patients with mCRC with a high NRG1 expression in the FIRE-1 trial. We did not detect a prognostic impact of HER2/neu and HER3 overexpression in MCRC with respect to PFS and OS.

\section{Acknowledgments}

The authors thank all patients and families, the FIRE-1 study centres and the medical staff who contributed to patient care.

This project was supported by DKTK, the German Cancer Consortium.

\section{Conflicts of interest}

D.P.M. received a research grant from the WeigandBohnewand-Gravenhorst-Fonds for this project. For the remaining authors there are no conflicts of interest. 


\section{References}

1 Douillard JY, Siena S, Cassidy J, Tabernero J, Burkes R, Barugel M, et al. Randomized, phase III trial of panitumumab with infusional fluorouracil, leucovorin, and oxaliplatin (FOLFOX4) versus FOLFOX4 alone as first-line treatment in patients with previously untreated metastatic colorectal cancer: the PRIME study. J Clin Oncol 2010; 28:4697-4705.

2 Van Cutsem E, Kohne CH, Lang I, Folprecht G, Nowacki MP, Cascinu S, et al. Cetuximab plus irinotecan, fluorouracil, and leucovorin as first-line treatment for metastatic colorectal cancer: updated analysis of overall survival according to tumor KRAS and BRAF mutation status. J Clin Oncol 2011; 29:2011-2019.

3 Leahy DJ. Structure and function of the epidermal growth factor (EGF/ErbB) family of receptors. Adv Protein Chem 2004; 68:1-27.

4 Pinkas-Kramarski R, Shelly M, Guarino BC, Wang LM, Lyass L, Alroy I, et al. ErbB tyrosine kinases and the two neuregulin families constitute a ligandreceptor network. Mol Cell Biol 1998; 18:6090-6101.

5 Kani K, Warren CM, Kaddis CS, Loo JA, Landgraf R. Oligomers of ERBB3 have two distinct interfaces that differ in their sensitivity to disruption by heregulin. J Biol Chem 2005; 280:8238-8247.

6 Hellyer NJ, Kim MS, Koland JG. Heregulin-dependent activation of phosphoinositide 3-kinase and Akt via the ErbB2/ErbB3 co-receptor. J Biol Chem 2001; 276:42153-42161.

7 Dawson JP, Berger MB, Lin CC, Schlessinger J, Lemmon MA, Ferguson KM. Epidermal growth factor receptor dimerization and activation require ligandinduced conformational changes in the dimer interface. Mol Cell Biol 2005; 25:7734-7742.

8 Berger MB, Mendrola JM, Lemmon MA. ErbB3/HER3 does not homodimerize upon neuregulin binding at the cell surface. FEBS Lett 2004; 569:332-336.

9 Rüschoff J, Hanna W, Bilous M, Hofmann M, Osamura RY, Penault-Llorca F et al. HER2 testing in gastric cancer: a practical approach. Mod Pathol 2012; 25:637-650.

10 Rüschoff J, Dietel M, Baretton G, Arbogast S, Walch A, Monges G, et al. HER2 diagnostics in gastric cancer-guideline validation and development of standardized immunohistochemical testing. Virchows Arch 2010; 457:299-307.

11 Gown AM, Goldstein LC, Barry TS, Kussick SJ, Kandalaft PL, Kim PM, et al. High concordance between immunohistochemistry and fluorescence in situ hybridization testing for HER2 status in breast cancer requires a normalized IHC scoring system. Mod Pathol 2008; 21:1271-1277.

12 Valtorta E, Martino C, Sartore-Bianchi A, Penaullt-Llorca F, Viale G, Risio M, et al. Assessment of a HER2 scoring system for colorectal cancer: results from a validation study. Mod Pathol 2015; 28:1481-1491.

13 Ingold Heppner B, Behrens HM, Balschun K, Haag J, Kruger S, Becker T, et al. HER2/neu testing in primary colorectal carcinoma. Br J Cancer 2014; 111:1977-1984.

14 Lee WS, Park YH, Lee JN, Baek JH, Lee TH, Ha SY. Comparison of HER2 expression between primary colorectal cancer and their corresponding metastases. Cancer Med 2014; 3:674-680.
15 Sithanandam G, Anderson LM. The ERBB3 receptor in cancer and cancer gene therapy. Cancer Gene Ther 2008; 15:413-448.

16 Beji A, Horst D, Engel J, Kirchner T, Ullrich A. Toward the prognostic significance and therapeutic potential of HER3 receptor tyrosine kinase in human colon cancer. Clin Cancer Res 2012; 18:956-968.

17 Scartozzi M, Mandolesi A, Giampieri R, Bittoni A, Pierantoni C, Zaniboni A, et al. The role of HER-3 expression in the prediction of clinical outcome for advanced colorectal cancer patients receiving irinotecan and cetuximab. Oncologist 2011; 16:53-60.

18 Lédel F, Hallstrom M, Ragnhammar P, Ohrling K, Edler D. HER3 expression in patients with primary colorectal cancer and corresponding lymph node metastases related to clinical outcome. Eur J Cancer 2014; 50:656-662.

19 De Boeck A, Pauwels P, Hensen K, Rummens JL, Westbroek W, Hendrix A, et al. Bone marrow-derived mesenchymal stem cells promote colorectal cancer progression through paracrine neuregulin 1/HER3 signalling. Gut 2013; 62:550-560.

20 Grivas PD, Antonacopoulou A, Tzelepi V, Sotiropoulou-Bonikou G, Kefalopoulou Z, Papavassiliou AG, et al. HER-3 in colorectal tumourigenesis: from mRNA levels through protein status to clinicopathologic relationships. Eur J Cancer 2007; 43:2602-2611.

21 Ho-Pun-Cheung A, Assenat E, Bascoul-Mollevi C, Bibeau F, BoissiereMichot F, Cellier D, et al. EGFR and HER3 mRNA expression levels predict distant metastases in locally advanced rectal cancer. Int J Cancer 2011 ; 128:2938-2946.

22 Lédel F, Stenstedt K, Hallstrom M, Ragnhammar P, Edler D. HER3 expression in primary colorectal cancer including corresponding metastases in lymph node and liver. Acta Oncol 2015; 54:480-486.

23 Seo AN, Kwak Y, Kim DW, Kang SB, Choe G, Kim WH, et al. HER2 status in colorectal cancer: its clinical significance and the relationship between HER2 gene amplification and expression. PloS one 2014; 9:e98528.

24 Kountourakis P, Pavlakis K, Psyrri A, Rontogianni D, Xiros N, Patsouris E, et al. Prognostic significance of HER3 and HER4 protein expression in colorectal adenocarcinomas. BMC cancer 2006; 6:46.

25 Uner A, Ebinc FA, Akyurek N, Unsal D, Mentes BB, Dursun A. Vascular endothelial growth factor, c-erbB-2 and c-erbB-3 expression in colorectal adenoma and adenocarcinoma. Exp Oncol 2005; 27:225-228.

26 Fischer von Weikersthal L, Schalhorn A, Stauch M, Quietzsch D, Maubach PA, Lambertz $\mathrm{H}$, et al. Phase III trial of irinotecan plus infusional 5 -fluorouracil/folinic acid versus irinotecan plus oxaliplatin as first-line treatment of advanced colorectal cancer. Eur J Cancer 2011; 47:206-214.

27 Stahler A, Heinemann V, Giessen-Jung C, Crispin A, Schalhorn A, Stintzing $S$, et al. Influence of mRNA expression of epiregulin and amphiregulin on outcome of patients with metastatic colorectal cancer treated with 5-FU/LV plus irinotecan or irinotecan plus oxaliplatin as first-line treatment (FIRE 1-trial). Int J Cancer 2016; 138:739-746.

28 Seo AN, Kwak Y, Kim WH, Kim DW, Kang SB, Choe G, et al. HER3 protein expression in relation to HER2 positivity in patients with primary colorectal cancer: clinical relevance and prognostic value. Virchows Arch 2015; 466:645-654. 